Maria Júlia Acedo 1

Vânia Aparecida da Costa 1

Newton Carlos Polimeno 1

Carmen Sílvia Bertuzzo 2

\section{Programa comunitário de hemoglobinopatias: abordagem populacional a partir de doadores de sangue de Bragança Paulista, São Paulo, Brasil}

\author{
Screening program for hemoglobinopathies \\ based on blood donors from Bragança Paulista, \\ São Paulo, Brazil
}

1 Laboratório Universitário de Análises Clínicas do Hospital Universitário São Francisco, Universidade São Francisco. Av. São Francisco de Assis 218, Bragança Paulista, SP 12916-900, Brasil. mjuliag@uol.com.br 2 Departamento de Genética Médica, Faculdade de Ciências Médicas, Universidade Estadual de Campinas.

Rua Tessália Vieira de Camargo 126, Campinas, SP 13081-970, Brasil. bertuzzo@unicamp.br

\begin{abstract}
Screening programs for hemoglobinopathies target different population groups such as neonates, students, pregnant women, military personnel, recruits, and blood donors. The feasibility and efficiency of these programs basically depend on the population's receptivity, which in turn is related to highly complex economic, psychological, and sociocultural factors. The purpose of this study was to evaluate the population of Bragança Paulista, São Paulo, based on results from the blood donors' group and to compare these results with those previously obtained in a group of students from the same city. The sample consisted of 1,846 donors who had given blood at the São Francisco University Hematology Center, Bragança Paulista, São Paulo, from October 1998 to April 1999. Analysis of the 1,846 donors identified 31 individuals who were hemoglobinopathy carriers (1.68\%). The result was quite similar to that obtained in the group of students and demonstrated that the two methods are similar in relation to detection of hemoglobinopathies; however, the blood donor approach is more practical.
\end{abstract}

Key words Hemoglobinopathies; Blood Donors; Genetics; Health Services

Resumo A alta freqüência de heterozigotos e a gravidade clínica dos homozigotos sempre encorajaram os programas de triagem populacional das hemoglobinopatias hereditárias. Os programas comunitários das hemoglobinopatias admitem diferentes formas de abordagem populacional: recém-nascidos, estudantes, gestantes, militares, doadores de sangue, recrutas, entre outros. O presente estudo, tem por objetivo avaliar a comunidade de Bragança Paulista pela abordagem dos doadores de sangue e comparar com os resultados encontrados pela abordagem por meio dos estudantes, feita anteriormente nessa mesma cidade. O estudo foi realizado em 1.846 doadores voluntários de sangue, no período de outubro de 1998 até abril de 1999, no Hemonúcleo da Universidade São Francisco na cidade de Bragança Paulista, interior de São Paulo. Dos 1.846 doadores analisados, encontramos 31 portadores de hemoglobinopatias (1,68\%). Esse índice é extremamente próximo do encontrado pela análise dos estudantes, mostrando que os dois meios de abordagem são similares em termos de detecção de hemoglobinopatias, mas o método de doadores de sangue é mais viável em termos práticos.

Palavras-chave Hemoglobinopatias; Doadores de Sangue; Genética; Serviços de Saúde 


\section{Introdução}

As hemoglobinopatias hereditárias são alteração genéticas extremamente freqüentes em nosso meio. Devido a isso, foram implantados vários programas de triagem populacional para a sua investigação e controle. Aliás, essa importância tem sido ressaltada em nível mundial, uma vez que a criação de programas comunitários nos países de Terceiro Mundo vem sendo estimulada por vários organismos internacionais, como a Organização Mundial da Saúde (WHO Community Control of Hereditary Anaemias, 1983), a Academia de Ciências do Terceiro Mundo (TWAS, 1986) e a Organização Pan-Americana da Saúde (OPS, 1987). Isso porque à medida que as doenças infecciosas e a desnutrição vão sendo controladas, as hemoglobinopatias vêm emergindo como um dos mais importantes problemas de saúde pública dos países em desenvolvimento.

No Brasil, os programas de triagem das hemoglobinopatias têm tido um grande impulso graças ao Serviço de Aconselhamento Genético em Alterações Hematológicas da Universidade Estadual de Campinas (UNICAMP), que tem a função de fomento à implantação de núcleos de assistência integral a portadores de alterações hematogenéticas em cidades brasileiras, de forma que os doentes, os heterozigotos e os casais de risco possam ser orientados no próprio município onde residem. Nesses programas, têm sido enfatizadas a participação voluntária dos indivíduos e a opção pelo aconselhamento genético dos afetados (Ramalho et al., 1999).

Assim, em 1988, Ramalho iniciou seus trabalhos nas comunidades, começando pela cidade de Araras, São Paulo (Teixeira \& Ramalho,1994). A comunidade foi abordada através de 2.209 gestantes em um período de 51 meses. Foram diagnosticados 445 indivíduos com alterações hereditárias, dentre os quais, 432 eram portadores de traços hemoglobínicos e 13 eram doentes com anemia hemolítica crônica. Foram detectados também, 16 casais de risco.

A segunda cidade avaliada foi Bragança Paulista, São Paulo (Compri et al., 1996), onde 1.118 estudantes e 53 parentes foram analisados. Detectou-se 47 indivíduos com alterações hereditárias da hemoglobina (4\%).

Um terceiro estudo comunitário foi realizado em Campinas, São Paulo (Paiva-e-Silva \& Ramalho, 1997), onde 9.196 doadores de sangue e 91 familiares foram examinados, diagnosticando-se 162 portadores de traços hemoglobínicos, três doentes e quatro casais de risco.

Do exposto, é fácil deduzir que os programas comunitários das hemoglobinopatias ad- mitem diferentes formas de abordagem populacional: recém-nascidos, estudantes, gestantes, militares, doadores de sangue, recrutas, entre outros. Como a abordagem por meio de estudantes já havia sido realizada na comunidade de Bragança Paulista, neste estudo realizamos a abordagem por meio de doadores de sangue nessa mesma comunidade e comparamos os resultados das duas abordagens.

\section{Casuística e métodos}

O estudo foi realizado em 1.846 doadores voluntários de sangue não consangüíneos, no período de outubro de 1998 a abril de 1999, no Hemonúcleo da Universidade São Francisco (USF), na cidade de Bragança Paulista, interior de São Paulo.

A investigação laboratorial foi realizada no Laboratório Universitário de Análises Clínicas do Hospital Universitário São Francisco da USF. As amostras de sangue venoso $(4,5 \mathrm{ml})$ foram colhidas em Vacutainers ${ }^{\circledR}$, com EDTA (sal disódico do ácido etilenodinitrotetracético) como anticoagulante, na concentração de $1,5 \mathrm{mg} / \mathrm{ml}$ (Dacie \& Lewis, 1984). Os dados hematológicos foram determinados com a utilização de um equipamento HemoCue ${ }^{\circledR}$, Blood Hemoglobin.

Realizou-se uma análise qualitativa das hemoglobinas pela corrida eletroforética do hemolisado em fitas de gel de agarose e tampão barbital 0,05M pH 8,6, por 20 minutos a $240 \mathrm{~V}$. A hemoglobina fetal foi dosada segundo Betke et al. (1959). Para a confirmação diagnóstica, utilizamos o teste de solubilidade (HbS) e a eletroforese em gel de ágar, $\mathrm{pH}$ ácido (HbC) (Ramalho, 1986).

\section{Resultados}

Dos 1.846 doadores analisados, encontramos 21 portadores do traço falciforme $(1,13 \%)$, seis do traço da hemoglobina C $(0,32 \%)$, dois portadores de Persistência Hereditária de Hemoglobina Fetal - PHHF $(0,1 \%)$, um da hemoglobina $\mathrm{J}(0,05 \%)$ e um doador portador de hemoglobina $\mathrm{D}(0,05 \%)$. No total, foram diagnosticados 31 portadores de hemoglobinopatias (1,68\%). Se considerarmos apenas os portadores de hemoglobinopatias, teremos: traço da hemoglobina S $(67,74 \%)$, traço da hemoglobina C $(19,35 \%)$, portador de PHHF $(6,45 \%)$, portador de hemoglobina J $(3,22 \%)$ e de hemoglobina D $(3,22 \%)$.

Enviou-se a todos os indivíduos onde se identificou a presença de uma hemoglobinopatia, carta convite para comparecerem ao Ser- 
viço de Aconselhamento Genético do Hemocentro da UNICAMP, e entrar em contato com a Professora Carmen Sílvia Bertuzzo para receber orientação genética. Dos indivíduos convocados, $93 \%$ compareceram ao serviço, sendo que poucos deixaram de comparecer.

Todos os indivíduos que compareceram ao serviço trouxeram seus familiares para exame. No entanto, nem sempre conseguimos analisar todo o núcleo familiar, pois alguns parentes moravam em outras cidades ou estados. Examinamos 118 familiares desses indivíduos portadores de hemoglobinopatias, e entre esses detectamos 55 outros portadores desse problema, sendo, portanto o número médio de pessoas examinadas a partir de cada doador heterozigoto igual a 4,06 .

Somando-se a análise dos doadores e dos parentes, temos um total de 1.964 exames realizados, com um total de 86 indivíduos portadores de hemoglobinopatias, dando um índice de positividade geral de $4,37 \%$.

\section{Discussão e conclusão}

Ao compararmos nossos dados com os obtidos por Compri et al. (1996), podemos fazer as seguintes considerações:

- Com relação à população abordada, em nenhum dos dois casos, estudantes e doadores, utiliza-se uma amostra representativa de todos os segmentos da comunidade, pois no caso dos estudantes os analfabetos são excluídos e, no caso dos doadores, os anêmicos são excluídos. Aliás, essa foi a razão de termos restringido nosso estudo às alterações estruturais da hemoglobina, uma vez que as síndromes talssêmicas geralmente são acompanhadas de anemia.

- Com relação ao fluxo de triagem, diferentemente dos estudantes, entre doadores o fluxo é contínuo e tem uma média mensal de exames maior (setenta no estudo em estudantes e 245 em doadores).

- A amostra de estudantes é mais trabalhosa de ser conseguida, uma vez que é necessário um contato prévio com a escola, diretor, professores, estudantes e pais. No caso de doadores, o acesso é individual e em nosso estudo todos os abordados concordaram em realizar os exames, o que não aconteceu com os estudantes $(55,4 \%)$.

Com isso, concluímos que na triagem de alterações estruturais de hemoglobinopatias, a abordagem por doadores de sangue apresenta maior viabilidade mantendo a eficiência, quando comparada à abordagem por estudantes (4\% em estudantes e $4,37 \%$ em doadores). Tal conclusão concorda com a observada por Ramalho et al. (1999). Além desse aspecto, convém salientar a importância da triagem das hemoglobinopatias em doadores de sangue, uma vez que vários receptores são homozigotos para essas alterações.

\section{Agradecimentos}

Os autores agradecem ao Hemonúcleo e ao Laboratório Universitário de Análises Clínicas da Universidade São Francisco pela colaboração prestada na realização deste trabalho. 


\section{Referências}

BETKE, K.; MARTI, H. R. \& SCHLICHT, I., 1959. Estimation of small percentage of foetal haemoglobin. Nature,184:1977-1978.

BOWMAN, J., 1991. Prenatal screening for hemoglobinopathies. Human Genetics, 48:433-438.

COMPRI, M. B.; POLIMENO, N. C.; STELLA, M. B. \& RAMALHO, A. S., 1996. Programa comunitário de hemoglobinopatias hereditárias em população estudantil brasileira. Revista de Saúde Pública, 30:187-195.

DACIE, J. V. \& LEWIS, S. M., 1984. Pratical Haematology. $6^{\text {th }}$ Ed. Edinburgh/London/Melbourne/New York: Churchill Livinsgstone.

OPS (Organización Panamericana de la Salud), 1987. Ejecución de Acciones de Salud en Genética - Informe de un Comité de Expertos en Genética Médica. Habana: OPS

PAIVA-E-SILVA, R. B. \& RAMALHO, A. S., 1997. Riscos e benefícios da triagem genética: $\mathrm{O}$ traço falciforme como modelo de estudo em uma questão brasileira. Cadernos de Saúde Pública, 13:285-294.

RAMALHO, A. S., 1986. As Hemoglobinopatias Hereditárias. Um Problema de Saúde Pública no Brasil. Ribeirão Preto: Editora da Sociedade Brasileira de Genética.
RAMALHO, A. S.; PAIVA-E-SILVA, R. B.; TEIXEIRA, R. C. \& COMPRI, M. B., 1999. Hemoglobin screening: Response of a Brazilian community to optional programs. Cadernos de Saúde Pública, 15:591595

TEIXEIRA, R. C. \& RAMALHO, A. S.,1994. Genetics and public health: Response of a Brazilian population to an optional hemoglobinopathy program. Revista Brasileira de Genética, 17:435-438.

TWAS (Third World Academy of Science), 1986. Final Report: South North Round Table on Haemoglobinopathies. Trieste: TWAS.

WHO COMMUNITY CONTROL OF HEREDITARY ANAEMIAS, 1983. Memorandum from a WHO meeting. Bulletin of the World Health Organization, 61:63-80.

Recebido em 29 de junho de 2001

Versão final reapresentada em 9 de janeiro de 2002 Aprovado em 9 de abril de 2002 